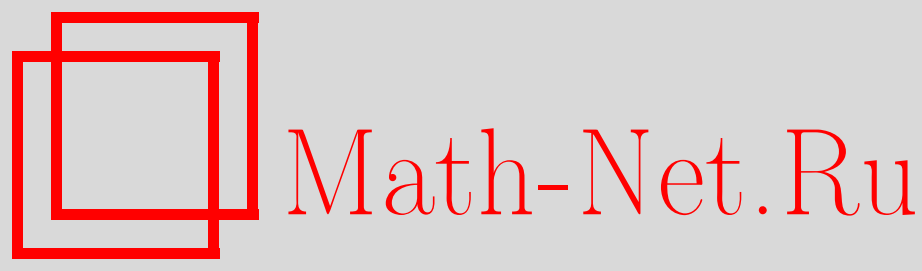

А. А. Хамзин, Р. Р. Нигматуллин, И. И. Попов, Микроскопическая модель недебаевской диэлектрической релаксации. Закон Коула-Коула и его обобщение, ТМФ, 2012, том 173, номер 2, 314-332

DOI: https://doi.org/10.4213/tmf8336

Использование Общероссийского математического портала Math-Net.Ru подразумевает, что вы прочитали и согласны с пользовательским соглашением http: //www . mathnet.ru/rus/agreement

Параметры загрузки:

IP : 3.93.64.190

26 апреля 2023 г., 05:43:27

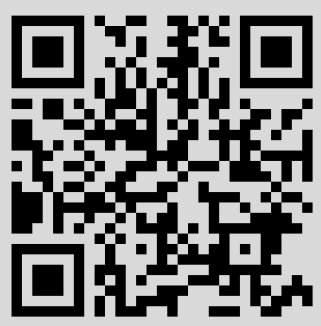




\title{
МИКРОСКОПИЧЕСКАЯ МОДЕЛЬ НЕДЕБАЕВСКОЙ ДИЭЛЕКТРИЧЕСКОЙ РЕЛАКСАЦИИ. ЗАКОН КОУЛА-КОУЛА И ЕГО ОБОБЩЕНИЕ
}

\begin{abstract}
Построена микроскопическая модель недебаевской (неэкспоненциальной) диэлектрической релаксации в сложных системах, которая базируется на самоподобной пространственно-временной организации релаксационного процесса. В рамках построенной модели приведен вывод выражения Коула-Коула для комплексной диэлектрической проницаемости и показано, что показатель $\alpha$, фигурирующий в этом выражении, равен фрактальной размерности пространственно-временного самоподобного ансамбля, характеризующего структуру среды и происходящий в ней процесс релаксации. Найдена связь макроскопического времени релаксации с микро- и мезопараметрами изучаемой системы. Получено обобщенное выражение Коула-Коула для комплексной диэлектрической проницаемости, содержащее логопериодические поправки, появляющиеся вследствие дискретной масштабной инвариантности фрактальной структуры, порождающей релаксационный процесс на мезоскопическом масштабе. Найденное выражение для диэлектрической проницаемости может быть использовано при интерпретации диэлектрических спектров в неупорядоченных диэлектриках.
\end{abstract}

Ключевые слова: диэлектрическая релаксация, комплексная диэлектрическая проницаемость, недебаевские диэлектрические спектры, фракталы, дискретная масштабная инвариантность, логопериодические осцилляции.

\section{1. ВВЕДЕНИЕ}

В последние годы наблюдается всестороннее исследование физики мягких конденсированных сред с целью изучения структуры, динамики и макроскопического поведения сложных систем (CC). СC - это широкий и достаточно разнообразный класс материалов, которые имеют в своей основе некристаллическое происхождение. Полимеры, биополимеры, коллоидные системы (эмульсии и микроэмульсии), биологические клетки, пористые материалы, а также жидкие кристаллы можно отнести

* Институт физики, Казанский (Приволжский) федеральный университет, Казань, Россия. E-mail: airat.khamzin@rambler.ru 
к классу СС. В значительном большинстве этих материалов диэлектрическая релаксация доминирует на мезоскопическом масштабе [1]. Простой экспоненциальный закон и классическая модель броуновской диффузии не могут описать релаксационные явления и кинетику СС. Неэкспоненциальный закон релаксации и аномальная диффузия - это те общие особенности, которые можно охарактеризовать термином "странная кинетика" [2].

Как правило, для полноценной характеристики релаксационных процессов в СС требуется привлечение различных методов, чтобы охватить соответствующие диапазоны частот. В этой связи диэлектрическая спектроскопия имеет свои преимущества, поскольку современные диэлектрические спектрометры позволяют охватить очень широкий диапазон частот (от $10^{-6}$ до $10^{11}$ Гц) и температур (от -170 до $\left.+500^{\circ} \mathrm{C}\right)[3]-[5]$.

Известно, что при воздействии внешнего электрического поля на диэлектрик поляризация материала достигает своего равновесного значения не мгновенно, а через определенный промежуток времени. Аналогично, когда внешнее поле выключается, поляризация распадается благодаря тепловому движению и характеризуется функцией затухания (спада) диэлектрической поляризации $\varphi(t)$ :

$$
\varphi(t)=\frac{P(t)}{P(0)},
$$

где $P(t)$ - модуль вектора поляризации единицы объема. Выражение для вектора диэлектрического смещения $\mathbf{D}(t)$ для случая зависящих от времени полей может быть записано в следующем виде [6], [7]:

$$
\mathbf{D}(t)=\varepsilon_{\infty} \mathbf{E}(t)+\int_{0}^{t} \frac{d \Phi\left(t^{\prime}\right)}{d t^{\prime}} \mathbf{E}\left(t-t^{\prime}\right) d t^{\prime} .
$$

Здесь $\mathbf{D}(t)=\mathbf{E}(t)+\mathbf{P}(t), \varepsilon_{\infty}-$ высокочастотный предел комплексной диэлектрической проницаемости (КДП) $\varepsilon(i \omega)$, а $\Phi(t)$ - функция диэлектрического отклика $\Phi(t)=\left(\varepsilon_{0}-\varepsilon_{\infty}\right)(1-\varphi(t))$, где $\varepsilon_{0}-$ низкочастотный предел КДП $\varepsilon(i \omega)$ в отсутствие проводимости. КДП $\varepsilon(i \omega)$ связана с релаксационной функцией $\varphi(t)$ простым соотношением [6], [7]:

$$
\hat{\varepsilon}(i \omega)=\frac{\varepsilon(i \omega)-\varepsilon_{\infty}}{\varepsilon_{0}-\varepsilon_{\infty}}=\hat{L}\left[-\frac{d \varphi(t)}{d t} ; i \omega\right]=1-i \omega \hat{L}[\varphi(t) ; i \omega],
$$

где $\hat{L}$ - оператор лаплас-образа, который для произвольной функции от времени определяется как

$$
\hat{L}[f(t) ; p]=\int_{0}^{\infty} f(t) e^{-p t} d t .
$$

Если $\varphi(t)=e^{-t / \tau}$, где $\tau$ - характерное время релаксации, то из (3) для нормированной КДП $\hat{\varepsilon}(i \omega)$ получаем закон Дебая

$$
\hat{\varepsilon}(i \omega)=\frac{1}{1+i \omega \tau} .
$$

Для большинства изучаемых систем выражение (5) для диэлектрической проницаемости недостаточно адекватно описывает экспериментальные результаты. Это 
обстоятельство привело к поиску эмпирических соотношений, которые формально принимают во внимание распределение времен релаксации. В самом общем виде недебаевское (неэкспоненциальное) диэлектрическое поведение может быть описано в терминах непрерывного распределения времен релаксации $g(\tau)$ [7]. Это означает, что КДП может быть представлена в виде

$$
\hat{\varepsilon}(i \omega)=\int_{0}^{\infty} \frac{g(\tau)}{1+i \omega \tau} d \tau, \quad \int_{0}^{\infty} g(\tau) d \tau=1 .
$$

Соответствующее выражение для релаксационной функции примет вид

$$
\varphi(t)=\int_{0}^{\infty} e^{-t / \tau} g(\tau) d \tau
$$

Если мнимая часть КДП является симметричной функцией, состоящей из одного пика, то недебаевские диэлектрические спектры могут быть описаны эмпирическим соотношением Коула-Коула [8]

$$
\hat{\varepsilon}(i \omega)=\frac{1}{1+(i \omega \tau)^{\alpha}}, \quad 0<\alpha<1,
$$

где $\alpha$ - эмпирический показатель, который обычно интерпретируется как мера уширения пика диэлектрических потерь.

В приближении линейного отклика флуктуации поляризации, вызванные тепловым движением, такие же, как макроскопические перестройки, индуцированные электрическим полем [9], [10]. Поэтому мы можем приравнять релаксационную функцию и макроскопическую дипольную корреляционную функцию (ДКФ) $\psi(t)$ :

$$
\varphi(t) \cong \psi(t)=\frac{\langle\mathbf{M}(t) \mathbf{M}(0)\rangle}{\langle\mathbf{M}(0) \mathbf{M}(0)\rangle},
$$

где $\mathbf{M}(t)$ - флуктуирующий дипольный момент единицы объема, который равен векторной сумме всех молекулярных дипольных моментов. Законы, регулирующие ДКФ, непосредственно связаны со структурными и кинетическими свойствами образца и характеризуют макроскопические свойства изучаемой системы. Таким образом, экспериментальные функции $\Phi(t)$ и $\varphi(t)$ или $\psi(t)$ могут быть использованы для получения информации о динамических свойствах изучаемого диэлектрика. Диэлектрическая релаксация многих СС отклоняется от простого экспоненциального закона и может быть описана законом Кольрауша-Вильямса-Уотса [11], [12]

$$
\varphi(t)=e^{-(t / \tau)^{\nu}}
$$

с характерным временем релаксации $\tau$ и эмпирическим показателем $0<\nu \leqslant 1$. Другой распространенный экспериментально наблюдаемый закон для ДКФ - это асимптотически степенной закон [13], [14]

$$
\varphi(t)=A\left(\frac{t}{\tau_{1}}\right)^{-\mu}, \quad t \geqslant \tau_{1},
$$


с амплитудой $A$, показателем $\mu$ и характеристическим временем $\tau_{1}$, которое связано с эффективным временем релаксации микроскопической структурной единицы. Выражения (10) и (11) для релаксационной функции показывают, что процесс релаксации в СС замедляется по сравнению с простым экспоненциальным законом. Возникает вопрос: в чем физическая причина замедления процесса релаксации? Ниже мы попытаемся ответить на этот вопрос.

Целью настоящей работы является формулировка физической модели, в рамках которой можно, во-первых, понять причины недебаевского поведения диэлектрической релаксации в СС и, во-вторых, получить выражение Коула-Коула (8) для КДП и тем самым придать физический смысл параметру $\alpha$, а также получить связь этого параметра и макроскопического времени релаксации $\tau$ с микроскопическими параметрами среды.

\section{2. КОНЦЕПЦИЯ ПАМЯТИ И ДРОБНАЯ КИНЕТИКА}

На наш взгляд, наиболее общий путь вычисления временны́х корреляционных функций (BКФ), к которым можно отнести и ДКФ (10), предлагается формализмом проекционных операторов Цванцига [15] и Мори [16], поскольку он исходит из первых принципов. В рамках указанного метода уравнение для ВКФ имеет вид [17]

$$
\frac{d \psi(t)}{d t}=-\int_{0}^{t} K\left(t-t^{\prime}\right) \psi\left(t^{\prime}\right) d t^{\prime}
$$

где $K(t)$ есть так называемая функция памяти, удовлетворяющая условию нормировки

$$
\tau \int_{0}^{\infty} K(t) d t=1
$$

причем параметр $\tau$ характеризует (не обязательно экспоненциальное) время релаксации. Если память в системе отсутствует (марковский процесс), то $K(t)=\tau^{-1} \delta(t)$ и мы вновь приходим к экспоненциальному поведению ВКФ. В реальной ситуации мы имеем дело с немарковскими процессами, когда в изучаемой системе присутствует память, что и приводит к неэкспоненциальному поведению ВКФ (в частности, ДКФ). Приведем уравнение (12) к удобному для дальнейшего изложения виду. Для этого проинтегрируем его от $t$ до $\infty$ и воспользуемся свойством (13):

$$
\psi(t)=\int_{0}^{t} M\left(t-t^{\prime}\right) \psi\left(t^{\prime}\right) d t^{\prime}+\tau^{-1} \int_{t}^{\infty} \psi\left(t^{\prime}\right) d t^{\prime},
$$

где функцию

$$
M(t)=\int_{t}^{\infty} K(u) d u
$$

назовем интегральной функиией памяти. При выводе уравнения (14) мы приняли во внимание принцип затухания корреляций: $\lim _{t \rightarrow \infty} \psi(t)=0$. Если рассматривать систему на временах $t>\tau$, что и будет предполагаться в дальнейшем, то второе слагаемое в правой части уравнения (14) мало по сравнению с первым, поэтому им можно пренебречь и записать уравнение (14) в виде

$$
\psi(t) \cong \int_{0}^{t} M\left(t-t^{\prime}\right) \psi\left(t^{\prime}\right) d t^{\prime}
$$


Теперь запишем кинетическое уравнение для релаксационной функции, которая приводит к эмпирическому выражению Коула-Коула (8) для КДП. Используя аппарат дробного интегро-дифференцирования и соотношение (3), кинетическое уравнение можно записать в виде

$$
\frac{d \varphi(t)}{d t}+\tau_{0}^{-\alpha} D_{t}^{1-\alpha} \varphi(t)=0
$$

где ${ }_{0} D_{t}^{1-\alpha}-$ оператор дробного дифференцирования Римана-Лиувилля [18]:

$$
{ }_{0} D_{t}^{1-\alpha} f(t)=\frac{1}{\Gamma(\alpha)} \frac{d}{d t} \int_{0}^{t} \frac{f\left(t^{\prime}\right)}{\left(t-t^{\prime}\right)^{1-\alpha}} d t^{\prime}, \quad 0 \leqslant \alpha<1 .
$$

Возможность построения такого дифференциального уравнения для релаксационной функции связана со следующим свойством оператора дробного дифференцирования [18]:

$$
\hat{L}\left[{ }_{0} D_{t}^{1-\alpha} f(t) ; i \omega\right]=(i \omega)^{1-\alpha} \hat{L}[f(t) ; i \omega] .
$$

Далее, проинтегрировав уравнение (17) от $t$ до $\infty$ и приняв во внимание, что

$$
\lim _{t \rightarrow \infty} \varphi(t)=0
$$

приведем его к виду

$$
\varphi(t)=-\tau_{0}^{-\alpha} D_{t}^{-\alpha} \varphi(t)
$$

где ${ }_{0} D_{t}^{-\alpha}-$ оператор дробного интегрирования Римана-Лиувилля [18]:

$$
{ }_{0} D_{t}^{-\alpha} f(t)=\frac{1}{\Gamma(\alpha)} \int_{0}^{t} \frac{f\left(t^{\prime}\right)}{\left(t-t^{\prime}\right)^{1-\alpha}} d t^{\prime} .
$$

Сравнивая уравнение (20) с уравнением (16), заключаем, что к закону Коула-Коула приводит следующее выражение для интегральной функции памяти:

$$
M(t)=-\frac{\tau^{-\alpha} t^{\alpha-1}}{\Gamma(\alpha)},
$$

или для самой функции памяти:

$$
K(t)=\frac{\tau^{-\alpha} t^{\alpha-2}}{\Gamma(\alpha-1)}
$$

Таким образом, базируясь на аппарате Мори-Цванцига и концепции памяти, мы заключаем, что природа неэкспоненциальной релаксации связана с наличием эффектов памяти, а именно с возможным степенным поведением функции памяти (23).

Чтобы продвинуться дальше в физической интерпретации параметра $\alpha$ в законе Коула-Коула, обратимся к физическому смыслу операции дробного интегрирования. Физическая интерпретация дробного интеграла с действительным показателем была предложена в работе [19] и также связывается с наличием памяти в изучаемой системе. Обобщение этого результата на случай комплексно-сопряженных показателей и более строгая формулировка прежнего результата были даны в работе [20]. 
Наличие памяти означает, что если в момент времени $t^{\prime}$ на систему действует сила $f\left(t^{\prime}\right)$, то возникает поток $J$, величина которого в последующий момент времени $t>t^{\prime}$ задается равенством

$$
J(t)=\int_{0}^{t} M\left(t-t^{\prime}\right) f\left(t^{\prime}\right) d t^{\prime}
$$

Пусть память в системе проявляется на бесконечном интервале времени, предшествующем времени $t$, но не во все моменты времени. Предположим, например, что память сохраняется только на малых отрезках времени, распределенных на множестве Кантора (канторовой "пыли") [21]. Тогда, проведя нетривиальные вычисления, мы приходим к следующей временно́й зависимости функции памяти [19], [20]:

$$
M\left(t-t^{\prime}\right) \sim[\sqrt{2}(1-\xi) t]^{-d} \frac{1}{\Gamma(d)}\left(t-t^{\prime}\right)^{d-1},
$$

где $d$ - фрактальная размерность множества Кантора, $\xi$ - параметр подобия множества Кантора [21]. Подставляя это выражение в равенство (24), приводим его к виду

$$
J(t)=[\sqrt{2}(1-\xi)]^{-d} D_{t}^{-d} f(t) .
$$

В результате показатель дробного интеграла равен фрактальной размерности множества Кантора и связан с мерой сохранения “остаточной” памяти.

Таким образом, появление оператора дробного интегрирования в физических уравнениях можно связать с самоподобным (фрактальным) характером процесса эволюции системы. Это заключение, в свою очередь, указывает на то, что причину появления дробных интегро-дифференциальных операторов в кинетических уравнениях для релаксационной функции следует искать в самоподобном характере процесса релаксации. Но прежде чем формулировать самоподобную модель релаксационного процесса, остановимся на некоторых важных для дальнейшего изложения положениях концепции фрактала.

Использование концепции фрактала в физике связывается со свойством масштабной инвариантности исследуемой среды как особого рода симметрии. Если $\Phi(x)-$ некоторая функция, характеризующая систему, то масштабная инвариантность системы определяется функциональным уравнением

$$
\Phi(\lambda x)=\gamma \Phi(x)
$$

где константа $\gamma$ показывает, как меняются свойства системы при изменении масштаба в $\lambda$ раз. Решением функционального уравнения (27) является степенная функция

$$
\Phi(x)=A x^{\nu},
$$

где $A$ - некоторая константа, а показатель степени $\nu=\ln \gamma / \ln \lambda$. Однако фракталы обладают свойством дискретной масштабной инвариантности, т. е. более низкой симметрией, чем масштабная инвариантность. Это означает, что функциональное уравнение (27) выполняется только при определенном специфическом выборе $\lambda$ 
(а также $\gamma$ ), а именно на бесконечном наборе дискретных значений $\lambda_{n}=\lambda^{n}$, где $\lambda-$ фундаментальный масштабный параметр.

Отметим, что поскольку $x \rightarrow \lambda x$ и $\Phi(x) \rightarrow \gamma^{-1} \Phi(\lambda x)$ эквивалентно преобразованию $y=\ln x \rightarrow y+\ln \lambda$ и $\ln \Phi(y) \rightarrow \ln \Phi(y+\ln \lambda)-\ln \gamma$, то масштабное преобразование сводится просто к трансляции $\ln x$, приводящей, в свою очередь, к трансляции $\ln \Phi(x)$. Таким образом, непрерывная масштабная инвариантность эквивалентна непрерывной трансляционной инвариантности для логарифмических переменных. Дискретная масштабная инвариантность в результате сводится к ограничению непрерывной трансляционной масштабной инвариантности. Это ограничение предполагает, что $\ln \Phi(x)$ просто транслируется при трансляции $\ln x$ на кратные $\ln \lambda$ величины. Таким образом, переход от непрерывной масштабной инвариантности к дискретной масштабной инвариантности можно сравнить (в логарифмическом масштабе) с переходом от жидкого состояния к твердому состоянию в физике конденсированных сред. В результате наличие дискретной масштабной инвариантности приводит к наложению на степенную зависимость (28) периодического в логарифмическом масштабе возмущения

$$
\Phi(x)=x^{\nu} W\left(\frac{\ln x}{\ln \lambda}\right),
$$

где $W(y)$ - некоторая периодическая функция с единичным периодом $(W(y+1)=$ $W(y))$, которую можно представить в виде ряда Фурье

$$
W(y)=\sum_{k=-\infty}^{\infty} c_{k} e^{2 \pi i k y}
$$

здесь $c_{k}$ - коэффициенты ряда Фурье, конкретные выражения для которых зависят от выбора функции $\Phi(x)$ (см. приложение). Подстановка выражения (30) в формулу (29) приводит ее к виду

$$
\Phi(x)=\sum_{k=-\infty}^{\infty} c_{k} x^{\nu+i k \Omega}
$$

где $\Omega=2 \pi / \ln \lambda$. Таким образом, понижение симметрии с непрерывной масштабной инвариантности до дискретной масштабной инвариантности приводит к возникновению спектра комплексных показателей для степенной зависимости.

\section{3. САМОПОДОБНАЯ МОДЕЛЬ ДИЭЛЕКТРИЧЕСКОЙ РЕЛАКСАЦИИ}

Следуя работе [22], представим рассматриваемую диэлектрическую среду в виде иерархически организованной совокупности дипольных кластеров. В неупорядоченных средах проявляется ближний порядок в расположении частиц (элементарных диполей), поэтому из ближайших диполей формируются кластеры первого уровня. Естественно, что между кластерами первого уровня присутствует определенная пространственная корреляция, что приводит к организации из этих кластеров первого 


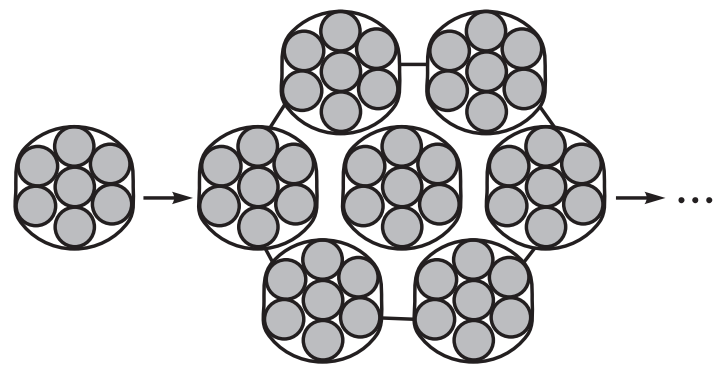

a

6

Рис. 1. Схематическое изображение самоподобной структуры диэлектрической среды: кластер $n$-го уровня (а), кластер $(n+1)$-го уровня (б).

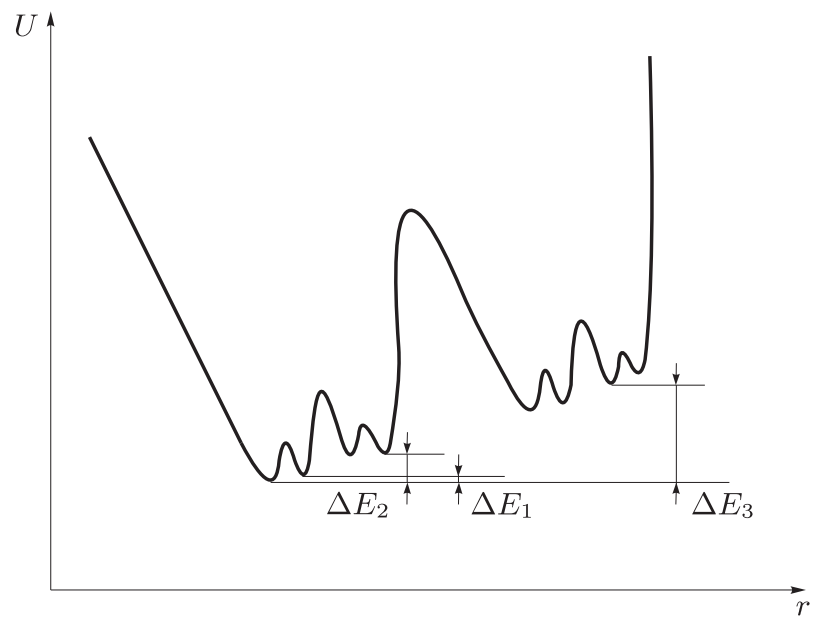

Рис. 2. Схематическое изображение ландшафта потенциального барьера во внешнем электрическом поле.

уровня кластеров второго уровня. С помощью такого самоподобного построения можно получить кластер $n$-го уровня (см. рис. 1 ).

Для анализа микроскопической кинетики диэлектрической релаксации данной иерархической структуры рассмотрим хорошо известную модель релаксатора Фрелиха [23]. В этой модели релаксатор находится в двух устойчивых точках равновесия, которые разделены потенциальным барьером. При действии внешнего поля появляется энергетическая разность $\Delta E$ между минимумами потенциального рельефа и система начинает процесс релаксации с характерным временем

$$
\tau=\tau_{0} e^{\Delta E / T} .
$$

Сделаем правомерное предположение, что каждый минимум, в свою очередь, имеет также два минимума, которые имеют подобную структуру потенциального 
рельефа (см. рис. 2). Такое самоподобное усложнение потенциального рельефа соответствует увеличению масштабов геометрической структуры, рассмотренной выше. Таким образом, мы получаем иерархию времен релаксации

$$
\tau_{1}<\tau_{2}<\tau_{3}<\cdots<\tau_{n}<\tau_{n+1}<\cdots<\tau_{n_{\max }}
$$

которая монотонно возрастает, поскольку и величины барьеров, и энергетическая разница между минимумами также возрастают. Это обстоятельство, в свою очередь, означает, что в системе идет множество релаксационных процессов, которые сменяют друг друга.

Базируясь на этой модели, рассмотрим релаксационную кинетику такой иерархической структуры. Предположим, что в некотором прошлом в системе была создана начальная поляризация, и отдельные частицы и кластеры частиц взаимодействуют посредством дипольных и мультипольных взаимодействий соответственно. Релаксационные процессы начинаются после того, как в начальный момент времени $t=0$ внешнее поле отключается. В первую очередь происходит релаксация на первом уровне (со временем $\tau_{1}$ ), это обусловлено тем, что элементарным диполям относительно легко преодолевать потенциальный барьер, который образуют соседние диполи. При этом релаксация на втором уровне еще не происходит, поскольку для кластера второго уровня вероятность преодолеть потенциальный барьер, образованный соседними кластерами, в которых большая часть диполей ориентирована в одном направлении, очень низкая. Когда на первом уровне отрелаксирует определенное количество диполей, только тогда на втором уровне начинается релаксация $\left(\right.$ со временем $\left.\tau_{2}\right)$. Таким образом, при ослаблении мультипольных корреляций данного кластера с его соседями возможен переход его в деполяризованное состояние как целого. Дальше процесс самоподобно повторяется. В рассмотренном процессе времена релаксации образуют иерархическое множество во временно́м пространстве по закону (33). Такая иерархически соподчиненная структура релаксации и является, на наш взгляд, основной причиной замедления релаксации, приводящей к трансформации дебаевской экспоненты в более медленно спадающие со временем зависимости (10) и (11).

Придадим рассмотренной физической модели соответствующую математическую формулировку. Пусть число диполей в кластере $l$-го уровня равно $N_{l}$, а время релаксации $\tau=\tau_{l}$. В соответствии с представленной иерархически соподчиненной организацией кластеров и соответствующих в них релаксационных процессов подчиним числа $N_{l}$ и $\tau_{l}$ следующим условиям самоподобия:

$$
N_{l}=N_{0} b^{l}, \quad \tau_{l}=\tau_{0} \xi^{l}, \quad-L_{\min } \leqslant l<L_{\max }, \quad L_{\min }, L_{\max } \gg 1,
$$

где $b>1, \xi>1$, а параметры $N_{0}$ и $\tau_{0}$ характеризуют мезоскопический масштаб системы. Отметим, что мы произвели изменение (по сравнению с (33)) в нумерации уровней самоподобного релаксационного процесса: $l=n-L_{\min }-1, n_{\max }=L_{\min }+$ $L_{\max }+1$. Это связано с выделением некоторого характерного (мезоскопического) масштаба, которому удобно сопоставить уровень $l=0$. 


\section{4. ЗАКОН КОУЛА-КОУЛА И ЕГО ОБОБЩЕНИЕ}

Если теперь произвести усреднение уравнения (16) по некоторому достаточно большому промежутку времени наблюдения $T \geqslant \tau_{\max }=\tau_{0} \xi^{L_{\max }}$ с функцией распределения времен релаксации $g(\tau)$, то получим уравнение для усредненной ДКФ (или релаксационной функции):

$$
\begin{gathered}
\bar{\psi}(t)=\int_{0}^{t} \bar{M}\left(t-t^{\prime}\right) \bar{\psi}\left(t^{\prime}\right) d t^{\prime} \\
\bar{\psi}(t)=\int \psi(t, \tau) g(\tau) d \tau, \quad \bar{M}(t)=\int M(t, \tau) g(\tau) d \tau .
\end{gathered}
$$

В соответствии с моделью (34) выберем функцию распределения времен релаксации $g(\tau)$ в виде

$$
g(\tau)=\sum_{l=-L_{\min }}^{L_{\max }} \frac{N_{l}}{N} \delta\left(\tau-\tau_{l}\right)
$$

Тогда в уравнении (35) усредненное (эффективное) выражение для интегральной функции памяти примет вид

$$
\bar{M}(\tau)=\sum_{l=-L_{\min }}^{L_{\max }} \frac{N_{l}}{N} M\left(t, \tau_{l}\right) .
$$

Нетрудно заметить, что интегральную функцию памяти $M(t, \tau)$ можно представить в виде

$$
M(t, \tau)=\frac{1}{\tau} G\left(\frac{t}{\tau}\right), \quad G(0)=1, \quad G(\infty)=0 .
$$

Поэтому с учетом (34) усредненное выражение для интегральной функции памяти (38) примет вид

$$
\bar{M}(\theta)=\sum_{l=-L_{\min }}^{L_{\max }} \frac{N_{l}}{N} \frac{1}{\tau_{l}} G\left(\frac{t}{\tau_{l}}\right)=c \tau_{0}^{-1} \sum_{l=-L_{\min }}^{L_{\max }} b^{l} \xi^{-l} G\left(\theta \xi^{-l}\right),
$$

где $c=N_{0} / N, \theta=t / \tau_{0}$. Легко проверить, что выражение для эффективной интегральной функции памяти (40) удовлетворяет (при $L_{\min }, L_{\max } \gg 1$ ) функциональному уравнению вида (27)

$$
\bar{M}(\xi \theta)=\frac{b}{\xi} \bar{M}(\theta) .
$$

Решение полученного функционального уравнения имеет вид (29):

$$
\bar{M}(\theta)=\theta^{d-1} W\left(\frac{\ln \theta}{\ln \xi}\right),
$$

где показатель степени

$$
d=\frac{\ln b}{\ln \xi}
$$

мы называем фрактальной размерностъю пространственно-временного самоподобного ансамбля, $W(y)$ - периодическая функция (30) с периодом, равным 1. 
Анализ выражения (40) показывает (см. приложение), что при $0<d<1$ коэффициенты Фурье $c_{k}$ имеют следующий вид:

$$
c_{k}=\frac{c \Omega}{2 \pi \tau_{0}} \mathfrak{G}(1-d-i \Omega k),
$$

где $\Omega=2 \pi / \ln \xi, \mathfrak{G}(s)$ - меллин-образ функции $G(\theta)$ :

$$
\mathfrak{G}(s)=\int_{0}^{\infty} G(\theta) \theta^{s-1} d \theta .
$$

Если пренебречь наличием логопериодических осцилляций, то эффективная интегральная функция памяти примет вид

$$
\bar{M}(t)=\frac{c \Omega}{2 \pi \tau_{0}^{d}} t^{d-1} \mathfrak{G}(1-d),
$$

который согласуется с выражением (22). Таким образом, можно сделать важный вывод, что параметр $\alpha$ в законе Коула-Коула совпадает с фрактальной размерностью $d$, определяемой выражением (43), а макроскопическое время релаксации $\tau$ имеет вид

$$
\tau^{-d}=-\frac{c \Omega}{2 \pi} \mathfrak{G}(1-d) \Gamma(d) \tau_{0}^{-d}
$$

Определим кинетическое уравнение и выражение для КДП в случае учета логопериодического возмущения. Для этого подставим выражение для усредненной интегральной функции памяти (42) в уравнение (35), получаем

$$
\begin{aligned}
\bar{\psi}(t) & =\int_{0}^{t} \bar{M}\left(t-t^{\prime}\right) \bar{\psi}\left(t^{\prime}\right) d t^{\prime}= \\
& =\frac{c \Omega}{2 \pi} \sum_{k=-\infty}^{\infty} \tau_{0}^{-(d+i \Omega k)} \mathfrak{G}(1-d-i \Omega k) \int_{0}^{t}\left(t-t^{\prime}\right)^{d+i \Omega k-1} \bar{\psi}\left(t^{\prime}\right) d t^{\prime}= \\
& =\frac{c \Omega}{2 \pi} \sum_{k=-\infty}^{\infty} \tau_{0}^{-(d+i \Omega k)} \mathfrak{G}(1-d-i \Omega k) \Gamma(d+i \Omega k)_{0} D_{t}^{-(d+i \Omega k)} \bar{\psi}(t) .
\end{aligned}
$$

Вводя обозначения

$$
d_{k}=d+i \Omega k, \quad \tau_{k}^{-d_{k}}=-\frac{c \Omega}{2 \pi} \mathfrak{G}\left(1-d_{k}\right) \Gamma\left(d_{k}\right) \tau_{0}^{-d_{k}}
$$

и дифференцируя обе части равенства, приведем уравнение (48) к виду

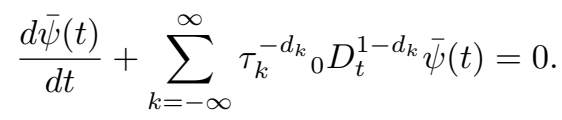

Полученное уравнение представляет собой кинетическое уравнение для ДКФ с учетом логопериодического возмущения, возникающего вследствие использования фрактальной модели релаксационного процесса, которая обладает свойством дискретной масштабной инвариантности. Осуществляя преобразование Лапласа уравнения (50), получим следующее выражение для лаплас-образа релаксационной функции:

$$
\hat{L}[\bar{\psi}(t) ; i \omega]=\left[i \omega+\sum_{k=-\infty}^{\infty} \tau_{k}^{-d_{k}}(i \omega)^{1-d_{k}}\right]^{-1}
$$


Подставляя полученное выражение в формулу (3), найдем выражение для нормированной КДП:

$$
\begin{aligned}
& \hat{\varepsilon}(i \omega)=\frac{\varepsilon(i \omega)-\varepsilon_{\infty}}{\varepsilon_{0}-\varepsilon_{\infty}}=1-i \omega \hat{L}[\bar{\psi}(t) ; i \omega]=\frac{1}{1+R(i \omega)}, \\
& R(i \omega)=\left[\sum_{k=-\infty}^{\infty} \tau_{k}^{-d_{k}}(i \omega)^{-d_{k}}\right]^{-1} .
\end{aligned}
$$

Таким образом, выражение (52) представляет собой обобщение закона Коула-Коула для КДП и учитывает логопериодические поправки, возникающие вследствие использования самоподобной концепции структурно-динамической организации неупорядоченной среды при построении модели релаксационного процесса в диэлектрике.

Обсудим вопрос о степени влияния логопериодических поправок на форму диэлектрического спектра. Приведем выражение для функции $R(i \omega)$ к виду

$$
R(i \omega)=(i \omega \tau)^{d}\left[1+2 \sum_{n=1}^{\infty}\left|a_{n}\right| \cos \left(\varphi_{n}+n \Omega \ln \left(i \omega \tau_{0}\right)\right)\right]^{-1}
$$

где

$$
a_{n}=\frac{\mathfrak{G}\left(1-d_{n}\right) \Gamma\left(d_{n}\right)}{\mathfrak{G}(1-d) \Gamma(d)}, \quad \varphi_{n}=\arg \left(a_{n}\right) .
$$

Анализ, проведенный для некоторых модельных представлений функции памяти, показывает, что коэффициенты $a_{k}$ с ростом $k$ по модулю очень быстро спадают. Действительно, если, например, выбрать функцию памяти $K(t)=\left(2 / \tau^{2} \sqrt{\pi}\right) e^{-(t / \tau)^{2}}$, которая переходит в дельта-функцию при $\tau \rightarrow 0$, то выражения для коэффициентов $a_{k}$ примут вид (см. приложение)

$$
a_{k}=\frac{\Gamma\left(1-d_{k} / 2\right) \Gamma\left(d_{k}-1\right)}{\Gamma(1-d / 2) \Gamma(d-1)},
$$

т. е. коэффициенты $a_{k}$ с ростом $k$ спадают по модулю как

$$
\left|a_{k}\right| \sim e^{-2.4 \Omega k}
$$

Поэтому в выражении для $R(i \omega)$ (52) можно с хорошей точностью ограничиться учетом только первой гармоники:

$$
R(i \omega) \approx(i \omega \tau)^{d}\left[1+2\left|a_{1}\right| \cos \left(\varphi_{1}+\Omega \ln \left(i \omega \tau_{0}\right)\right)\right]^{-1} .
$$

Тогда выражение для нормированной КДП примет вид

$$
\hat{\varepsilon}(i \omega) \approx\left[1+\frac{(i \omega \tau)^{d}}{1+2\left|a_{1}\right| \cos \left(\varphi_{1}+\Omega \ln \left(i \omega \tau_{0}\right)\right)}\right]^{-1} .
$$

Покажем, что выражение (53) переходит в формулу Дебая при $d \rightarrow 1$. Введем в рассмотрение среднее время релаксации $\bar{\tau}$, определяемое формулой

$$
\frac{1}{\bar{\tau}}=\sum_{l=-L_{\min }}^{L_{\max }} \frac{N_{l}}{N} \tau_{l}^{-1}=\frac{c}{\tau_{0}} \sum_{l=-L_{\min }}^{L_{\max }} \xi^{-(1-d) l}=\frac{c}{\tau_{0}} \frac{\xi^{(1-d) L_{\min }}-\xi^{-(1-d)\left(L_{\max }+1\right)}}{1-\xi^{-(1-d)}},
$$



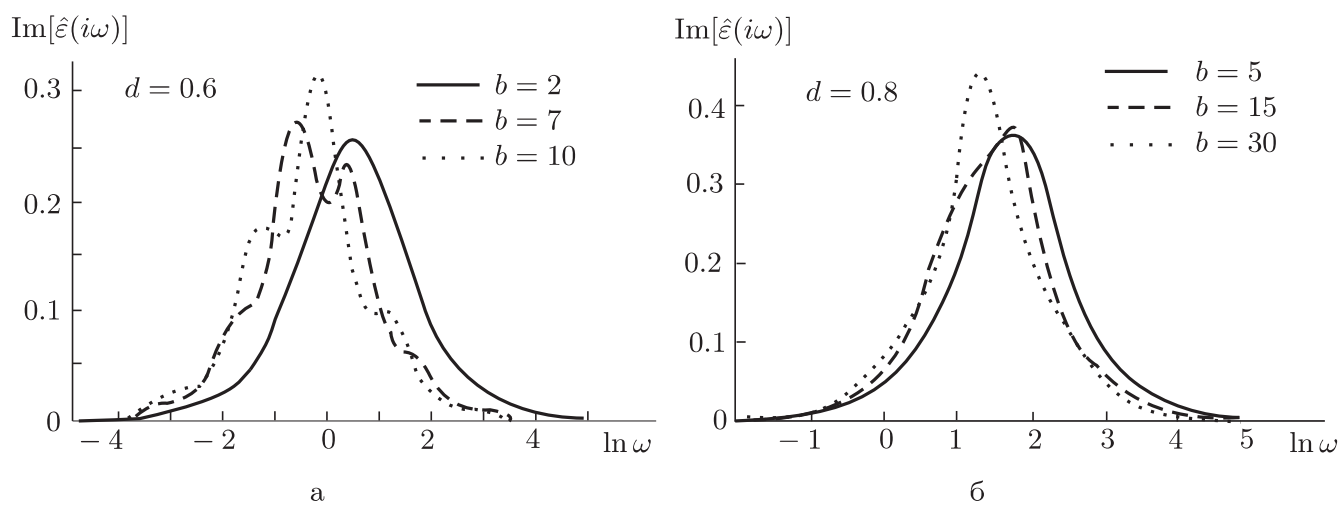

Рис. 3. Частотная зависимость $\operatorname{Im}[\hat{\varepsilon}(i \omega)]$, где $\hat{\varepsilon}(i \omega)$ определяется выражением (53) при $\tau_{0}=10^{-5}, c=10^{-3}$ для различных значений параметров $d$ и $b$.

и воспользуемся условием нормировки

$$
\sum_{l=-L_{\min }}^{L_{\max }} \frac{N_{l}}{N}=c \sum_{l=-L_{\min }}^{L_{\max }} \xi^{d l}=c \frac{\xi^{d\left(L_{\max }+1\right)}-\xi^{-d L_{\min }}}{\xi^{d}-1}=1
$$

Уравнения (59) и (60) можно рассматривать как уравнения для определения $L_{\min }$, $L_{\max }$, если определить среднее время $\bar{\tau}$ в качестве дополнительного параметра модели. Исключая из уравнений (59) и (60) $L_{\min }, L_{\max }$, при $L_{\min } \gg 1$ получим следующую приближенную связь между параметрами $\bar{\tau}$ и $\tau_{0}$ :

$$
\tau_{0} \simeq \bar{\tau} c^{1 / d} \frac{\left(\xi^{d}-1\right)^{1-1 / d}}{\xi^{d-1}-1}
$$

откуда при $d \rightarrow 1$ найдем

$$
\tau_{0} \rightarrow \frac{c}{(d-1) \ln \xi} \bar{\tau}
$$

Учитывая, что при $d \rightarrow 1+0$, как это следует из (47) и $(61), \tau \rightarrow \bar{\tau}$, поскольку $\lim _{s \rightarrow 0} s \mathfrak{G}(s)=\mathfrak{G}(0)=1$ и $a_{k} \rightarrow 0(k \neq 0)$, из (53) немедленно воспроизводится закон Дебая со временем релаксации, равным среднему времени релаксации $\bar{\tau}$.

Выражение для КДП с учетом логопериодических поправок (52) (или (58)) дает возможность по-новому взглянуть на интерпретацию диэлектрических спектров в СС. Действительно, на рис. 3 приведены графики для мнимой составляющей нормированной КДП, определяемой выражением (52) при некоторых значениях параметров $\tau_{0}, c, d, b$ и $K(t)=\left(2 / \tau^{2} \sqrt{\pi}\right) e^{-(t / \tau)^{2}}$.

Из рис. 3 видно, что учет логопериодического возмущения приводит к наложению на кривую Коула-Коула осцилляций, амплитуда которых при заданной функции 
памяти определяется параметрами $d$ и $b$. Наличие осцилляций приводит к возникновению особой многопиковой структуры мнимой части нормированной КДП в частотной области (см. рис. За). Но такое поведение мнимой части КДП в частотной области не стоит связывать с часто наблюдаемой в экспериментах многопиковой структурой диэлектрических спектров, как, например, в спектрах с $\beta$-релаксацией [24], [25]. Здесь дополнительные пики появляются на более высоких частотах по сравнению с основным пиком и, по-видимому, связаны с появлением в системе релаксационных процессов другой природы. Если осцилляции и не проявляются ярко, как, например, на рис. 3б, то их учет приводит к возникновению явной асимметрии в области пика потерь, которая отсутствовала в случае стандартной эмпирической кривой Коула-Коула.

\section{5. ЗАКЛЮЧЕНИЕ}

Базируясь на фрактальной модели диэлектрической релаксации, мы вывели эмпирическое выражение Коула-Коула для КДП, а также получили его обобщение, содержащее логопериодические поправки, обусловленные свойством дискретной масштабной инвариантности пространственно-временной структуры неупорядоченной среды. Эти результаты обобщают результаты работ одного из авторов, полученные ранее в рамках нестрогого подхода [23], [26].

Полученное выражение для параметра $\alpha$ раскрывает, в определенном смысле, его физическое содержание. А именно, этот параметр равен фрактальной размерности самоподобного пространственно-временного ансамбля, которая является основной характеристикой релаксационного процесса и связана со структурными параметрами $b$ и $\xi$ среды. Нами найдена связь макроскопического времени релаксации $\tau$ с мезоскопическим временем релаксации $\tau_{0}$ и со структурными параметрами $b$ и $\xi$. Это обстоятельство, в свою очередь, позволит из экспериментальных результатов получать информацию о структурных параметрах диэлектрической среды и релаксационных явлениях в ней на мезоскопическом масштабе. Первые предварительные результаты, связанные с влиянием логопериодических осцилляций, нашли свое экспериментальное подтверждение при описании процесса диэлектрической релаксации в реакции полимеризации винилпирролидона в работе [27]. Использование концепции фрактала при построении релаксационного процесса в диэлектрике приводит к необходимости учета логопериодического возмущения, возникающего вследствие дискретной масштабной инвариантности фрактала. Эти осцилляции в зависимости от их интенсивности могут внести асимметрию вблизи пика потерь или привести к более существенным его искажениям, как показанно на рис. За. Возможность такого типа поведения диэлектрических спектров можно использовать при интерпретации диэлектрических данных в широком классе неупорядоченных систем, обладающих самоподобным пространственно-временным поведением.

ПРИЛОЖЕНИЕ Расчет усредненной интегральной функции памяти $\bar{M}(\theta)$

Определяя фрактальную размерность $d$ (43) в качестве независимого параметра модели вместо параметра $b$, представим выражение для усредненной интегральной 
функции памяти (40) в виде

$$
\bar{M}(\theta)=c \tau_{0}^{-1} \sum_{l=-L_{\min }}^{L_{\max }} \xi^{(d-1) l} G\left(\theta \xi^{-l}\right) .
$$

Для расчета выражения (П.1) удобно осуществить преобразование Меллина:

$$
\begin{gathered}
\bar{M}(\theta) \stackrel{\mathrm{MT}}{=} \mathfrak{M}(s)=\int_{0}^{\infty} \bar{M}(\theta) \theta^{s-1} d \theta, \quad G(\theta) \stackrel{\mathrm{MT}}{=} \mathfrak{G}(s)=\int_{0}^{\infty} G(\theta) \theta^{s-1} d \theta, \\
\sigma_{1}<\operatorname{Re} s<\sigma_{2} .
\end{gathered}
$$

Тогда, учитывая свойство преобразования Меллина $G(a \theta) \stackrel{\mathrm{MT}}{=} a^{-s} \mathfrak{G}(s)$, из (П.1) получим

$$
\begin{gathered}
\mathfrak{M}(s)=c \tau_{0}^{-1} \mathfrak{G}(s) \sum_{l=-L_{\min }}^{L_{\max }} \xi^{(s+d-1) l} \simeq \frac{c \tau_{0}^{-1} \mathfrak{G}(s) \xi^{(s+d-1) L_{\max }}}{1-\xi^{-(s+d-1)}} \\
\max \left(\sigma_{1}, 1-d\right)<\gamma=\operatorname{Re} s<\sigma_{2}
\end{gathered}
$$

где мы приняли во внимание, что $L_{\min } \gg 1$. Осуществим обратное преобразование Меллина в (П.2) и, используя основную теорему о вычетах, получаем

$$
\bar{M}(\theta)=\frac{1}{2 \pi i} \int_{\gamma-i \infty}^{\gamma+i \infty} \mathfrak{M}(s) \theta^{-s} d s= \pm c \tau_{0}^{-1} \sum_{k} \operatorname{Res}_{s_{k}}\left[\frac{\mathfrak{G}(s) \xi^{(s+d-1) L_{\max }} \theta^{-s}}{1-\xi^{-(s+d-1)}}\right]
$$

где линию интегрирования мы замкнули на контур, при этом знак плюс соответствует левому контуру $\left(L_{+}\right)$, а знак минус - правому $\left(L_{-}\right), s_{k}-$ полюсы функции $\mathfrak{M}(s)$, попадающие в соответствующие контуры.

Для проведения дальнейших вычислений конкретизируем вид функции памяти $K(t)$. При выборе конкретного модельного представления для функции памяти будем руководствоваться следующими соображениями. Как уже упоминалось выше, отсутствие памяти в процессе релаксации соответствует выбору функции памяти в виде дельта-функции $\left(K(t)=\tau^{-1} \delta(t)\right)$. Учет эффектов памяти с математической точки зрения приводит к необходимости трансформации дельта-функции, например в некоторую "колоколообразную" функцию с конечной шириной. В качестве такой функции можно выбрать гауссовскую функцию, поскольку известно, что

$$
\delta(t)=\frac{2}{\sqrt{\pi}} \lim _{\tau \rightarrow 0} \frac{1}{\tau} e^{-t^{2} / \tau^{2}}
$$

Поэтому для демонстрации конкретных вычислений мы выбираем функцию памяти в виде

$$
K(t)=\frac{1}{\sqrt{\pi}} \frac{1}{\tau^{2}} e^{-t^{2} / \tau^{2}}
$$

Для данного вида функции памяти интегральная функция памяти (15) примет вид

$$
M(t)=\frac{1}{\tau} \operatorname{Erfc}\left(\frac{t}{\tau}\right)
$$




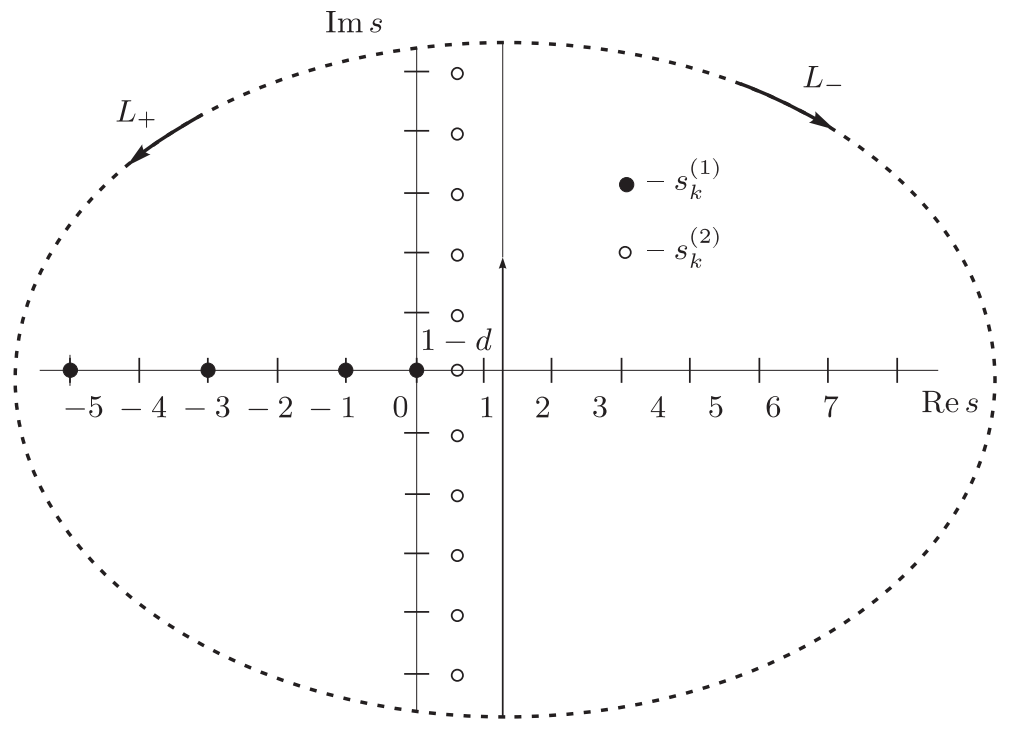

Рис. 4. Схема расположения полюсов функции $\mathfrak{M}(s)(П .5)$ и контуров $L_{ \pm}$ в комплексной плоскости.

где $\operatorname{Erfc}(x)$ - дополнительная функция ошибок. Преобразование Меллина функции $G(\theta)=\operatorname{Erfc}(\theta)$ дает

$$
\mathfrak{G}(s)=\frac{1}{\sqrt{\pi}} s^{-1} \Gamma\left(\frac{s+1}{2}\right), \quad \operatorname{Re} s>0 .
$$

В результате меллин-образ усредненной интегральной функции памяти принимает вид

$$
\mathfrak{M}(s)=\frac{c \tau_{0}^{-1}}{\sqrt{\pi}} s^{-1} \Gamma\left(\frac{s+1}{2}\right) \frac{\xi^{(s+d-1) L_{\max }}}{1-\xi^{-(s+d-1)}}, \quad \text { Re } s>1-d .
$$

Полюсы функции (П.5), которые являются простыми, можно разделить на две группы:

$$
s_{k}^{(1)}=0,-2 k-1, \quad k=0,1,2, \ldots,
$$

- полюсы функции $\mathfrak{G}(s)(\Pi .4)$,

$$
s_{k}^{(2)}=1-d+i \Omega k, \quad k \in \mathbb{Z},
$$

- полюсы функции $\xi^{(s+d-1) L_{\max }}\left[1-\xi^{-(s+d-1)}\right]^{-1}$. Если выбрать контур $L_{+}$, то в него попадают полностью полюсы обеих групп (см. рис. 4), и мы получаем из (П.3) следующий результат:

$$
\bar{M}(\theta)=\frac{c \tau_{0}^{-1}}{\sqrt{\pi}} \sum_{k=-\infty}^{\infty} \operatorname{Res}_{1-d+i \Omega k}\left[\frac{\Gamma((s+1) / 2) \xi^{(s+d-1) L_{\max } \theta^{-s}}}{s\left(1-\xi^{-(s+d-1)}\right)}\right]+
$$




$$
\begin{aligned}
& +\frac{c \tau_{0}^{-1}}{\sqrt{\pi}} \sum_{k=0}^{\infty} \operatorname{Res}_{-2 k-1}\left[\frac{\Gamma((s+1) / 2) \xi^{(s+d-1) L_{\max } \theta^{-s}}}{s\left(1-\xi^{-(s+d-1)}\right)}\right]+ \\
& +\frac{c \tau_{0}^{-1}}{\sqrt{\pi}} \operatorname{Res}_{0}\left[\frac{\Gamma((s+1) / 2) \xi^{(s+d-1) L_{\max } \theta^{-s}}}{s\left(1-\xi^{-(s+d-1)}\right)}\right] .
\end{aligned}
$$

Принимая во внимание, что полюсы простые и нет пересечения полюсов между группами, а также формулы

$$
\begin{aligned}
\underset{1-d+i \Omega k}{\operatorname{Res}}\left[\frac{f(s)}{1-\xi^{-(s+d-1)}}\right] & =\frac{f(1-d+i \Omega k)}{\ln \xi}, \\
\underset{-2 k-1}{\operatorname{Res}}\left[f(s) \Gamma\left(\frac{s+1}{2}\right)\right] & =\frac{2(-1)^{k} f(-2 k-1)}{k !},
\end{aligned}
$$

приводим выражение (П.6) к виду

$$
\begin{aligned}
\bar{M}(\theta)= & \frac{c \Omega \tau_{0}^{-1}}{2 \pi \sqrt{\pi}} \sum_{k=-\infty}^{\infty} \frac{\Gamma((2-d+i \Omega k) / 2) \theta^{-(1-d+i \Omega k)}}{1-d+i \Omega k}+ \\
& +\frac{2 c \tau_{0}^{-1}}{\sqrt{\pi}} \sum_{k=0}^{\infty} \frac{(-1)^{k+1} \xi^{-(2 k+2-d) L_{\max } \theta^{2 k+1}}}{k !(2 k+1)\left(1-\xi^{2 k+2-d}\right)}+c \tau_{0}^{-1} \frac{\xi^{-(1-d) L_{\max }}}{1-\xi^{1-d}} .
\end{aligned}
$$

Отметим, что первое слагаемое в (П.7) не зависит от параметра обрезания $L_{\max }$. Принимая во внимание условия $L_{\max } \gg 1,0<d<1$ и $\xi>1$, замечаем, что в (П.7) второе и третье слагаемые малы, поскольку в этом случае $\xi^{-(2 k+2-d) L_{\max }} \ll 1$, $\xi^{-(1-d) L_{\max }} \ll 1$, поэтому ими пренебрегаем. В результате получаем

$$
\bar{M}(\theta)=\frac{c \Omega \tau_{0}^{-1}}{2 \pi \sqrt{\pi}} \theta^{-(1-d)} \sum_{k=-\infty}^{\infty} \frac{\Gamma((2-d+i \Omega k) / 2)}{1-d+i \Omega k} \theta^{-i \Omega k} .
$$

Если выбрать контур $L_{-}$, то замечаем (см. рис. 4), что в него не попадает ни один полюс из обеих групп, поэтому здесь $\bar{M}(\theta) \equiv 0$. Полученное значение для усредненной интегральной функции памяти, очевидно, является аналитическим продолжением выражения (П.7) в область $\theta \leqslant 0$, поскольку выражение (П.7) существует при $\theta>0$.

Таким образом, для конкретного вида функции памяти мы показали, что усредненное выражение для интегральной функции памяти имеет вид (42), поскольку, как нетрудно видеть, выражение (П.8) легко приводится к виду (42), где функция $W(y)$ определяется в (30) и

$$
c_{k}=\frac{c \Omega \tau_{0}^{-1}}{2 \pi \sqrt{\pi}} \frac{\Gamma((2-d-i \Omega k) / 2)}{1-d-i \Omega k} .
$$

Проведенные выше вычисления для конкретного модельного представления функции памяти носили лишь демонстративный характер, их целью было показать возможность вычисления конкретных выражений для коэффициентов Фурье логопериодической функции $W(\ln \theta / \ln \xi)$. Предлагаемая схема расчета усредненной интегральной функции памяти, естественно, не ограничивается данным выбором функции памяти и может быть с успехом применена и для других модельных представлений функции памяти. Более того, вычисления, подобные приведенным выше, 
могут быть проведены и в общем случае, т. е. для общего модельного представления функции $K(t)$, поскольку для всех физически реализуемых модельных представлений функции памяти меллин-образ функции $G(\theta)$ принадлежит классу гипергеометрических функций:

$$
\mathfrak{G}(s)=\frac{\prod_{j=1}^{m} \Gamma\left(a_{j}+\alpha_{j} s\right) \prod_{j=1}^{n} \Gamma\left(b_{j}-\beta_{j} s\right)}{\prod_{j=1}^{p} \Gamma\left(c_{j}+\gamma_{j} s\right) \prod_{j=1}^{q} \Gamma\left(d_{j}-\delta_{j} s\right)},
$$

где параметры $a_{j}, b_{j}, c_{j}, d_{j} \in \mathbb{R}, \alpha_{j}, \beta_{j}, \gamma_{j}, \delta_{j} \in \mathbb{R}_{+}$, причем полюсы функции (П.10) не пересекаются и отделимы от полюсов $s_{k}^{(2)}$. Принимая этот факт во внимание, мы можем обобщить выражение (П.9) для усредненной интегральной функции памяти и представить коэффициенты Фурье логопериодической функции $W(\ln \theta / \ln \xi)$ в виде (45).

Благодарности. Работа выполнена в рамках тематического плана "Диэлектрическая спектроскопия и кинетика сложных систем" (бюджет 12-18 020210021000018 ).

\section{Список литературы}

[1] Y. Feldman, A. Puzenko, Ya. Ryabov, "Dielectric relaxation phenomena in complex materials", Advances in Chemical Physics, 133, eds. Y. P. Kalmykov, W. T. Coffey, S. A. Rice, Wiley, New York, 2006, 1-125.

[2] M. F. Shlesinger, G. M. Zaslavsky, J. Klafter, Nature, 363:6424 (1993), 31-37.

[3] A. Schönhals, F. Kremer, A. Hofmann, E. W. Fischer, E. Schlosser, Phys. Rev. Lett., 70:22 (1993), 3459-3462.

[4] P. Lunkenheimer, A. Pimenov, M. Dressel, Yu. G. Goncharov, R. Bohmer, A. Loidl, Phys. Rev. Lett., 77:2 (1996), 318-321.

[5] U. Scheider, P. Lunkenheimer, R. Brand, A. Loidl, J. Non-Cryst. Solids, 235-237 (1998), $173-179$.

[6] Г. Фрелих, Теория диэлектриков. Диэлектрическая проницаемость и диэлектрические потери, ИЛ, М., 1960.

[7] C. J.F. Böttcher, P. Bordewijk, Theory of Electric Polarization, v. 2: Dielectrics in Time-Dependent Fields, Elsevier, Amsterdam, 1992.

[8] K. S. Cole, R. H. Cole, J. Chem. Phys., 9:4 (1941), 341-351.

[9] G. Williams, Chem. Rev., 72:1 (1972), 55-69.

[10] R. H. Cole, "Dielectric polarization and relaxation", Molecular Liquids: Dynamics and Interactions, NATO ASI Series C, 135, eds. A. J. Barnes, W. J. Orville-Thomas, J. Yarwood, Reidel, Dordrecht, 1984, 59-110.

[11] R. Kohlrausch, Ann. Phys. (Leipzig), 12 (1847), 393.

[12] G. Williams, D. C. Watts, Trans. Faraday Soc., 66 (1970), 80-85.

[13] A. K. Jonscher, Dielectric Relaxation in Solids, Chelsea Dielectric Press, London, 1983.

[14] A. K. Jonscher, Universal Relaxation Law, Chelsea Dielectric Press, London, 1996.

[15] R. W. Zwanzig, "Statistical mechanics of irreversibility", Lectures in Theoretical Physics, 3, eds. W. E. Brittin, B. W. Downs, J. Downs, Interscience, New York, 1961, 106-141.

[16] H. Mori, Prog. Theor. Phys., 34:3 (1965), 399-416.

[17] J. P. Boon, S. Yip, Molecular Hydrodynamics, McGraw-Hill, New York, 1980.

[18] С. Г. Самко, А. А. Килбас, О. И. Маричев, Интеграль и производные дробного порядка и некоторые их приложения, Наука и техника, Минск, 1987.

[19] Р. Р. Нигматуллин, ТМФ, 90:3 (1992), 354-368. 
[20] R. R. Nigmatullin, A. Le Mehaute, J. Non-Cryst. Solids, 351:33-36 (2005), 2888-2899.

[21] Е. Федер, Фракталы, Мир, М., 1991.

[22] V. V. Novikov, V. P. Privalko, Phys. Rev. E, 64:3 (2001), 031504, 11 pp.

[23] R. R. Nigmatullin, Physica A, 363:2 (2006), 282-298.

[24] G. P. Johari, M. Goldstein, J. Chem. Phys., 53:6 (1970), 2372-2388.

[25] G. P. Johari, Ann. NY Acad. Sci., 279 (1976), 117-140.

[26] R. R. Nigmatullin, Physica B, 358:1-4 (2005), 201-205.

[27] R. R. Nigmatullin, A. A. Arbuzov, F. Salehli, A. Giz, I. Bayrak, H. Catalgil-Giz, Physica B, 388:1-2 (2007), 418-434.

Поступила в редакцию 19.03.2012, после доработки 23.04.2012 